\title{
Padrões de distribuição e abundância de gastrópodes límnicos no município de Acaraú, Ceará: avaliando aspectos da Teoria da Biogeografia de Ilhas
}

\author{
Marcos Roberto Santos * \\ Rafaela Camargo Maia \\ Instituto Federal de Educação, Ciência e Tecnologia do Ceará \\ Avenida Desembargador Armando de Sales Louzada, s/n, CEP 62580-000, Acaraú - CE, Brasil \\ * Autor para correspondência \\ marcosrob20@gmail.com
}

Submetido em 04/06/2018

Aceito para publicação em 04/10/2018

\section{Resumo}

Na Teoria da Biogeografia de Ilhas, a relação espécies-área e o isolamento espacial podem influenciar na distribuição de espécies nos habitat. Nesse contexto, ecossistemas lênticos são utilizados para fornecer informações sobre mecanismos locais de diversidade de organismos, entre eles, moluscos. O presente estudo avaliou os aspectos ecológicos e biogeográficos de moluscos límnicos verificando a influência do tamanho e distância dos corpos hídricos na distribuição das espécies. A amostragem foi realizada em lagoas, classificada por perímetro com diferentes distâncias entre elas, em Acaraú, Ceará. Foram capturados 2.067 gastrópodes, distribuídos em seis famílias, seis gêneros e nove espécies. Foram coletados os planorbídeos Biomphalaria glabrata e Drepanotrema cimex, que ainda não haviam sido registrados para o Ceará. Não foram observadas diferenças significativas na riqueza, abundância e diversidade em relação ao tamanho das lagoas. Entretanto, há uma tendência de maiores índices em lagoas médias, o que pode ser resultante de distúrbios intermediários. Também não foram observadas similaridades na estrutura da comunidade entre corpos de água próximos, o que pode ser explicado pela facilidade de dispersão das espécies devido à enchente em períodos chuvosos. Os dados aqui apresentados podem ser importantes para compreensão dos mecanismos responsáveis pelos padrões de distribuição de moluscos límnicos no nordeste brasileiro.

Palavras-chave: Ambientes lênticos; Malacofauna; Riqueza

\section{Abstract}

Distribution patterns and abundance of limnic gastropods in the municipality of Acaraú, Ceará: evaluating aspects of the Theory of Island Biogeography. According to the Theory of Island Biogeography, the relation species to area and spatial isolation can influence the distribution of species in habitats. Thus, lentic ecosystems are used to provide information on local mechanisms of diversity of organisms, including mollusks. The present study evaluated the ecological and biogeographic aspects of limnic mollusks by assessing the influence of the size and distance of water bodies on the distribution of species. Sampling was carried out in lagoons, classified by perimeter and with different distances, in Acaraú, Ceará. A total of 2,067 gastropods were collected; they were distributed in six families, six genera and nine species and included the planorbids 
Biomphalaria glabrata and Drepanotrema cimex, which had not yet been recorded for Ceará. No significant differences were observed in richness, abundance and diversity in relation to the size of the lagoons. However, there was a tendency for higher indices in medium-sized ponds, which may be due to intermediate disturbances. Similarities in community structure between nearby water bodies were not observed, which can be explained by the ease of dispersion of species due to flooding during rainy periods. The data presented here may be important for understanding the mechanisms responsible for the distribution patterns of limnic mollusks in northeastern Brazil.

Key words: Lentic environments; Mollusca fauna; Richness

\section{Introdução}

A biogeografia é um ramo das ciências naturais que se empenha em documentar e compreender os modelos espaciais de biodiversidade, os processos responsáveis pela distribuição dos organismos no espaço e sua mudança através do tempo (RECODER, 2011). Sendo assim, é a ciência que estuda a distribuição dos organismos em seus habitat, tanto no passado quanto no presente, incluindo padrões de variação ocorridos no planeta, relacionados à quantidade e aos tipos de seres vivos existentes nos mais diferentes ecossistemas, sejam marinho, terrestre ou de água doce (CARVALHO; ALMEIDA, 2011).

De acordo com a Teoria da Biogeografia de Ilhas, desenvolvida por MacArthur e Wilson (1967), vários fatores podem influenciar na distribuição e riqueza dos habitat. O fator mais relevante e difundido para explicar esses padrões é a relação espécies-área, na qual a riqueza de espécies aumenta progressivamente com o aumento da área (LOPES; CALIMAN 2008). Essa relação é parcialmente oriunda do efeito da influência do tamanho do habitat no tamanho das populações e no número de micro-habitat dentro dos ecossistemas (DODSON, 1992).

Outro fator é o isolamento espacial de comunidades, que exerce grande influência sobre a dispersão de organismos entre diferentes comunidades, tendo assim um efeito sobre a diversidade (GILLUNG, 2011). Segundo Srivastava et al. (2004) e De Meester et al. (2005), um maior grau de isolamento reduz a taxa de colonização de novos indivíduos e espécies para uma comunidade, diminuindo, portanto, a riqueza específica. Dentro desse contexto, ecossistemas aquáticos lênticos, como lagos, poças e açudes, podem ser importantes sistemas modelo na compreensão dos mecanismos responsáveis pelos padrões de distribuição da diversidade (LOPES; CALIMAN, 2008).

Ambientes aquáticos lênticos apresentam características como, por exemplo, fronteiras bem definidas na paisagem, evidenciando os ecótonos, ampla heterogeneidade espaço-temporal e maior suscetibilidade a distúrbios (GILLER, 2004). Dessa forma, possuem grande variedade de organismos que apresentam ciclos de vida curtos e maiores taxas de especiação, já que a água permite maior força de interações diretas e indiretas entre as espécies e maior conectividade entre microhabitat (GILLER, 2004). Dessa forma, esses ambientes permitem isolar e entender mais rapidamente o papel individual e interativo de mecanismos-chave como dispersão, especiação, extinções, fatores ambientais e interações bióticas na determinação dos padrões geográficos da diversidade (CARPENTER, 2001).

O Filo Mollusca é o terceiro maior do Reino Animal (AMARAL et al., 2005; KARDONG, 2011). Porém, apenas gastrópodes e bivalves ocupam os ambientes aquáticos límnicos (SIMONE, 2006), habitando preferencialmente o sedimento e a vegetação adjacente (LOPES-PITONI, 2001).

Esses organismos têm participação relevante nos ecossistemas que habitam, desempenhando importante papel na cadeia trófica, sendo utilizados como alimento por peixes, anfíbios, répteis, aves e mamíferos, entre eles o homem (DRÜGG-HAHN, 1997). Algumas espécies são hospedeiras intermediárias de várias doenças de interesse médico-veterinário (FRANÇA et al., 2007). Entretanto, há carência de estudos com moluscos de água doce (SIMONE, 2013), especialmente no nordeste do país.

Dessa forma, os objetivos deste estudo foram: i) caracterizar a comunidade de moluscos avaliando 
a composição da comunidade, riqueza de espécies, abundância de organismos e a diversidade biológica; ii) verificar o efeito dos tamanhos das lagoas na ocorrência das espécies de moluscos; e iii) analisar a distância entre as lagoas como fator de controle para a dissimilaridade das comunidades de moluscos límnicos.

\section{Material e Métodos}

\section{Área de estudo}

O estudo foi desenvolvido entre os meses de julho e agosto de 2016, em nove corpos de água doce na cidade Acaraú, estado do Ceará, localizada a $238 \mathrm{~km}$ de Fortaleza, capital do estado (Figura 1). A região apresenta clima tropical, temperatura média de $27,7^{\circ} \mathrm{C}$ e pluviosidade média anual de 1203 mm (CLIMATE-DATA.ORG, 2017).
As áreas de coleta foram escolhidas seguindo uma linha de semelhanças entre elas, sendo selecionados ambientes aquáticos lênticos, perenes, com mesmo nível de ocupação antrópica nas margens (habitações) e vegetação aquática composta por Eleocharis interstincta (Vahl) Roem \& Schult, 1994, Nymphaea amazonum Mart. \& Zucc, 1832 e Nymphoides grayana (Griseb.) Kuntze, 1969.

\section{Procedimentos de campo}

As lagoas amostradas foram classificadas de acordo com o perímetro, obedecendo a três categorias: Lagoas Pequenas (1-500 m), Médias (501-1500 m) e Grandes ( $\geq 1501 \mathrm{~m}$ ), com distância geográfica variável entre elas (Tabela 1). Os pontos de amostragem foram escolhidos de forma aleatória e variaram entre as lagoas para que locais de grande extensão não fossem subamostrados.

O tempo de coleta foi padronizado de acordo com a extensão do perímetro da lagoa, sendo estipulado um

FIGURA 1: Mapa da região amostral indicando a localização geográfica dos pontos de coleta em Acaraú, Ceará.

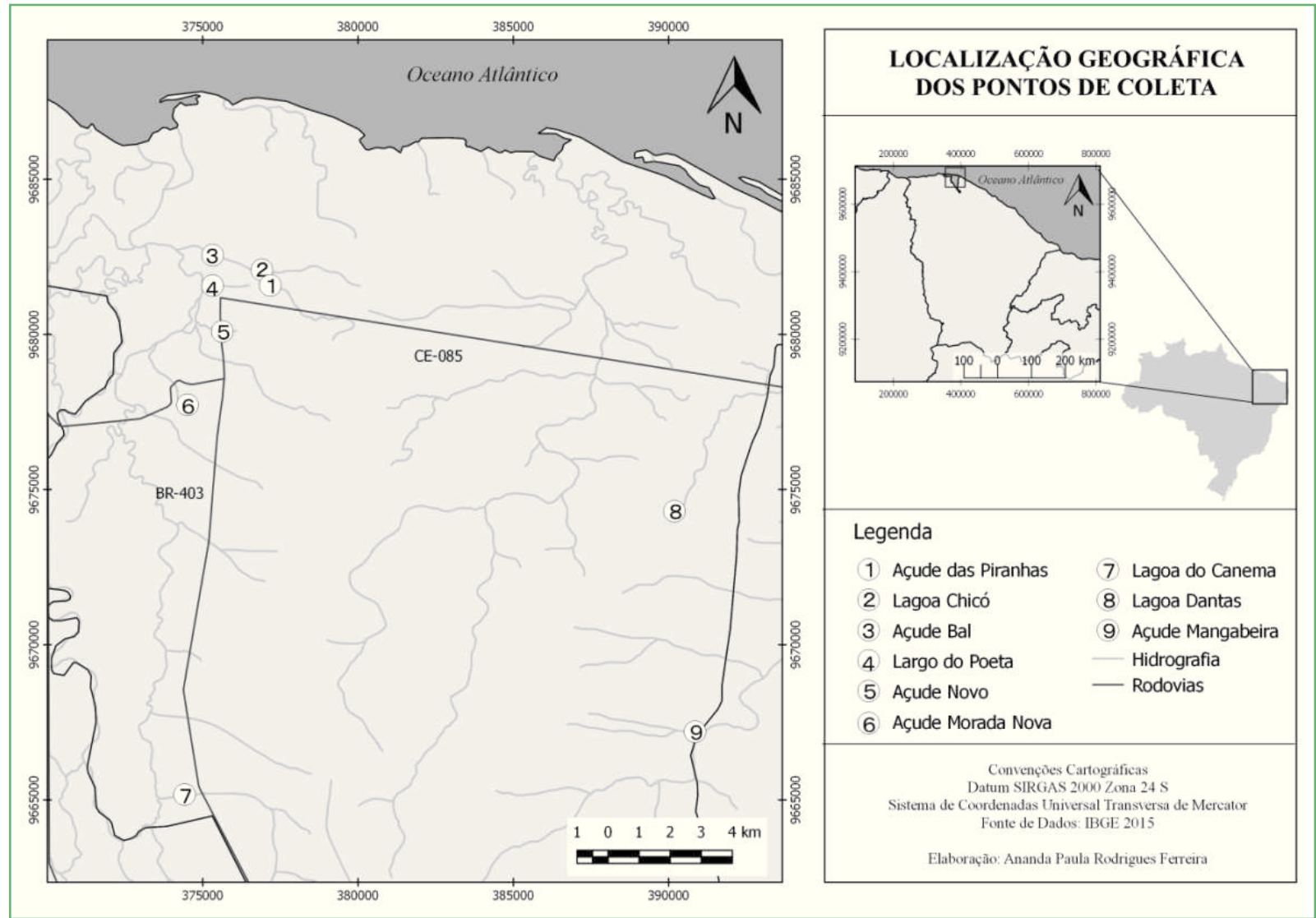


TABELA 1: Relação das distâncias entre as lagoas amostradas em Acaraú, Ceará, indicando o perímetro (m) e a classificação adotada quanto ao tamanho (Pequenas - P; Médias - M; Grandes - G).

\begin{tabular}{|c|c|c|c|c|c|c|c|c|c|c|c|}
\hline \multirow[b]{2}{*}{ Local } & \multirow[b]{2}{*}{$\begin{array}{l}\text { Perímetro } \\
\text { (m) }\end{array}$} & \multirow[b]{2}{*}{$\begin{array}{c}\text { Classificação } \\
\text { (P, M, G) }\end{array}$} & \multicolumn{9}{|c|}{ Distância (m) } \\
\hline & & & $\begin{array}{c}\text { Açude das } \\
\text { Piranhas }\end{array}$ & $\begin{array}{l}\text { Lagoa } \\
\text { Chicó }\end{array}$ & $\begin{array}{c}\text { Açude } \\
\text { Bal }\end{array}$ & $\begin{array}{c}\text { Largo do } \\
\text { Poeta }\end{array}$ & $\begin{array}{l}\text { Açude } \\
\text { Novo }\end{array}$ & $\begin{array}{l}\text { Açude } \\
\text { Morada } \\
\text { Nova }\end{array}$ & $\begin{array}{l}\text { Lagoa do } \\
\text { Canema }\end{array}$ & $\begin{array}{l}\text { Lagoa } \\
\text { Dantas }\end{array}$ & $\begin{array}{c}\text { Açude } \\
\text { Mangabeira }\end{array}$ \\
\hline $\begin{array}{l}\text { Açude das } \\
\text { Piranhas }\end{array}$ & $4.657,7$ & G & $\mathrm{X}$ & 56,5 & 785,3 & $1.478,5$ & $2.389,3$ & $4.977,4$ & $14.722,8$ & $13.340,6$ & $14.704,6$ \\
\hline Lagoa Chicó & 106 & $\mathrm{P}$ & 56,5 & X & 708,8 & $1.406,9$ & $2.376,3$ & $4.998,5$ & $14.721,5$ & $13.399,6$ & $14.774,1$ \\
\hline Açude Bal & $1.789,1$ & G & 785,3 & 708,8 & $\mathrm{X}$ & 864,2 & $2.394,6$ & $5.087,0$ & $14.132,5$ & $14.128,6$ & $15.505,9$ \\
\hline Largo do Poeta & 196 & $\mathrm{P}$ & $1.478,5$ & $1.406,9$ & 864,2 & $X$ & $1.509,6$ & $4.195,9$ & $14.067,8$ & $14.577,3$ & $15.752,1$ \\
\hline Açude Novo & 226,4 & $\mathrm{P}$ & $2.389,3$ & $2.376,3$ & $2.394,6$ & $1.509,6$ & $\mathrm{X}$ & $2.556,4$ & $12.498,5$ & $14.289,5$ & $15.155,5$ \\
\hline $\begin{array}{c}\text { Açude Morada } \\
\text { Nova }\end{array}$ & $1.210,6$ & M & $4.977,4$ & $4.998,5$ & $5.087,0$ & $4.195,9$ & $2.556,4$ & $\mathrm{X}$ & $9.761,9$ & $14.726,9$ & $15.055,5$ \\
\hline $\begin{array}{l}\text { Lagoa do } \\
\text { Canema }\end{array}$ & $1.306,4$ & M & $14.722,8$ & $14.721,5$ & $14.953,9$ & $14.067,8$ & $12.498,5$ & $9.761,9$ & $\mathrm{X}$ & $18.655,0$ & $17.221,7$ \\
\hline Lagoa Dantas & 659,9 & $\mathrm{M}$ & $13.340,6$ & $13.399,6$ & $14.128,6$ & $14.577,3$ & $14.289,5$ & $14.726,9$ & $18.655,0$ & $\mathrm{X}$ & $2.973,4$ \\
\hline $\begin{array}{c}\text { Açude } \\
\text { Mangabeira }\end{array}$ & $3.457,2$ & G & $14.704,6$ & $14.774,1$ & $15.505,9$ & $15.752,1$ & $15.155,5$ & $15.055,5$ & $17.221,7$ & $2.973,4$ & $\mathrm{X}$ \\
\hline
\end{tabular}

total de 15 minutos para cada ponto amostral marcado. Dessa forma, em lagoas pequenas foi estabelecido apenas um ponto para a realização de uma amostragem de 15 minutos. Em lagoas consideradas médias foram três pontos, totalizando 45 minutos para coleta. Nas lagoas grandes foram oito pontos, perfazendo 120 minutos para realização da coleta. As amostragens foram feitas sempre próximas à vegetação aquática, a uma profundidade máxima de $1 \mathrm{~m}$.

A busca pelos moluscos nas áreas de coleta foi realizada de modo direto por quatro pessoas divididas em duplas: dois pesquisaram a vegetação e o substrato, com o auxílio da concha de captura de moluscos, e dois capturaram os moluscos nos locais em que a concha não é indicada, como em superfícies duras (SANTOS et al., 2010). A concha de captura consistia em um cabo de madeira com aproximadamente $1 \mathrm{~m}$ de comprimento, acoplado a uma peneira com malha de $2 \mathrm{~mm}$.

Os moluscos coletados (Licença permanente para coleta de material zoológico número: 45584-1) em cada ponto foram acondicionados em sacos plásticos, com pequena quantidade de água do local de coleta para a manutenção da umidade, e identificados. Em laboratório, foram anestesiados em solução de $30 \mathrm{~g}$ de Cloreto de Magnésio $\left(\mathrm{MgCl}_{2}\right)$ diluído em $200 \mathrm{~mL}$ de água e a seguir fixados em álcool $70 \%$.

Para identificação, os exemplares foram analisados quanto a sua anatomia externa e, quando necessário, foi realizada a dissecção dos animais. O material testemunho foi depositado na Coleção Malacológica Prof. Henry Ramos Matthews da Universidade Federal do Ceará (Fortaleza).

\section{Análise de dados}

A riqueza e a abundância de moluscos entre lagoas/tamanho foram avaliadas com uma Análise de Variância (ANOVA). Quando detectadas diferenças entre as médias, ao nível de significância de 5\% ( $p<0,05)$, o teste de comparações múltiplas de Tukey foi aplicado. 
O padrão de variação na estrutura das comunidades da malacofauna foi avaliado com Análise de Agrupamento (Cluster) a partir do índice de similaridade de BrayCurtis, usando o programa PRIMER v6 (CLARKE; GORLEY, 2006). Essas análises consideraram os valores de densidade relativa das espécies. Também por meio desse programa foram realizadas a Análise de Similaridade (ANOSIM) e a Percentagem de Similaridade (SIMPER), visando detectar variações espaciais entre as lagoas.

Para avaliar a diversidade, foram utilizados o Índice de Simpson (POOLE, 1974), a Equabilidade de Pielou e o Índice de Shannon (LUDWIG; REYNOLDS, 1988).

\section{Resultados}

Foram coletados 2.067 moluscos, todos da Classe Gastropoda, distribuídos em seis famílias, sete gêneros e nove espécies. São elas: Melanoides tuberculatus (Thiaridae) (Müller, 1774); Biomphalaria straminea (Planorbidae) (Dunker, 1848); Biomphalaria glabrata (Planorbidae) (Say, 1818); Drepanotrema cimex (Planorbidae) (Moricand, 1839); Streptartemon molaris (Streptaxidae) Simone \& Casati, 2013; Pomacea lineata (Ampullariidae) (Spix, 1827); Ferrisia sp. (Ancylidae); Gundlachia sp. (Ancylidae); Omalonyx sp. (Succineidae).

TABELA 2: Riqueza e abundância dos moluscos límnicos amostrados nas lagoas de tamanhos pequeno (P), médio (M) e grande $(\mathrm{G})$ em Acaraú, Ceará.

\begin{tabular}{|c|c|c|c|c|}
\hline Local & Tamanho & Famílias & Abundância & Espécies \\
\hline \multirow{2}{*}{ Lagoa Chicó } & \multirow{2}{*}{$\mathrm{P}$} & Ancylidae & 2 & Ferrisia sp. \\
\hline & & Ampullariidae & 17 & Pomacea lineata \\
\hline \multirow{4}{*}{ Largo do Poeta } & \multirow{4}{*}{$\mathrm{P}$} & \multirow{2}{*}{ Planorbidae } & 110 & Drepanotrema cimex \\
\hline & & & 8 & Biomphalaria straminea \\
\hline & & Streptaxidae & 1 & Streptartemon molaris \\
\hline & & Ampullariidae & 18 & Pomacea lineata \\
\hline \multirow{2}{*}{ Açude Novo } & \multirow{2}{*}{$\mathrm{P}$} & Ampullariidae & 14 & Pomacea lineata \\
\hline & & Planorbidae & 2 & Biomphalaria straminea \\
\hline \multirow{3}{*}{ Lagoa Dantas } & \multirow{3}{*}{ M } & Ampullariidae & 255 & Pomacea lineata \\
\hline & & Planorbidae & 371 & Biomphalaria straminea \\
\hline & & Thiaridae & 452 & Melanoides tuberculatus \\
\hline \multirow{4}{*}{ Morada Nova } & \multirow{4}{*}{$\mathrm{M}$} & Ancylidae & 2 & Ferrisia sp. \\
\hline & & Ampullariidae & 23 & Pomacea lineata \\
\hline & & \multirow{2}{*}{ Planorbidae } & 3 & Drepanotrema cimex \\
\hline & & & 17 & Biomphalaria glabrata \\
\hline \multirow{2}{*}{ Lagoa do Canema } & \multirow{2}{*}{ M } & Ampullariidae & 4 & Pomacea lineata \\
\hline & & Planorbidae & 3 & Biomphalaria straminea \\
\hline \multirow{2}{*}{ Açude do Bal } & \multirow{2}{*}{ G } & Ampullariidae & 82 & Pomacea lineata \\
\hline & & Thiaridae & 316 & Melanoides tuberculatus \\
\hline \multirow{2}{*}{ Açude Mangabeira } & \multirow{2}{*}{$\mathrm{G}$} & Ampullariidae & 48 & Pomacea lineata \\
\hline & & Planorbidae & 27 & Biomphalaria straminea \\
\hline \multirow{5}{*}{ Açude das Piranhas } & \multirow{5}{*}{$\mathrm{G}$} & Ampullariidae & 76 & Pomacea lineata \\
\hline & & Thiaridae & 195 & Melanoides tuberculatus \\
\hline & & \multirow{2}{*}{ Ancylidae } & 7 & Ferrisia sp. \\
\hline & & & 3 & Gundlachia sp. \\
\hline & & Succineidae & 11 & Omalonyx sp. \\
\hline
\end{tabular}




\section{Efeito do tamanho das lagoas na ocorrência das espécies de moluscos}

A riqueza de espécies de moluscos encontrada nas lagoas de diferentes tamanhos durante o presente estudo está expressa na Tabela 2. Pomacea lineata está presente em todos os corpos hídricos, seguido por B. straminea, que está em cinco das nove lagoas pesquisadas. Melanoides tuberculatus e Ferrisia sp. estão presentes em três locais. Drepanotrema cimex foi coletado em dois corpos hídricos e B. glabrata, $S$. molaris, Omalonyx sp., Gundlachia sp. ficaram restritos a uma das localidades.

Não foram observadas diferenças significativas na riqueza de espécies de moluscos entre os tamanhos de lagoas amostrados $\left(\mathrm{F}_{2,6}=0,06250 ; \mathrm{p}=0,94002\right)$. As Lagoas Grandes e Médias apresentaram riqueza de seis espécies, pertencentes a quatro famílias, enquanto as Lagoas Pequenas apresentaram cinco espécies, representadas por três famílias.

Também não foram observadas diferenças significativas na abundância de moluscos entre os tamanhos das lagoas amostradas $\left(\mathrm{F}_{2,6}=0,58278\right.$, $\mathrm{p}=0,58709$ ). Entretanto, a maior abundância absoluta foi observada nas lagoas médias, com 1.130 organismos, seguido pelas lagoas grandes, com 765 organismos.
As lagoas pequenas apresentaram a menor abundância de espécies, com 172 moluscos amostrados.

Assim, a Lagoa Dantas apresentou maior abundância dos moluscos $P$. lineata, $B$. straminea e $M$. tuberculatus. Porém, $P$. lineata teve ampla distribuição, estando presente em todos os locais de amostragem, enquanto M. tuberculatus foi coletado somente no Açude Bal e no Açude das Piranhas. Biomphalaria straminea ocorreu no Largo do Poeta, no Açude Novo, na Lagoa do Canema e no Açude Mangabeira, em menor quantidade.

No Largo do Poeta foi coletado o único exemplar de $S$. molaris amostrado pelo presente trabalho. Essa área também teve a maior abundância de $D$. cimex, espécie também coletada no Açude Morada Nova, em menor número. Omalonyx sp. e Gundlachia sp. só foram amostrados no Açude das Piranhas. Nessa localidade foi coletado o maior número de Ferrisia sp., amostrada também na Lagoa Chicó e no Açude Morada Nova, onde a espécie foi pouco representada. Biomphalaria glabrata só ocorreu no Açude Morada Nova.

Os índices de diversidade adotados neste estudo para expressar a diversidade dos moluscos límnicos indicam que as lagoas amostradas apresentam diversidade equivalente (Tabela 3), diferenciando-se umas das outras pela abundância de uma ou duas espécies (Figura 2).

FIGURA 2: Distribuição da abundância dos moluscos límnicos amostrados nas lagoas de tamanhos pequeno (P), médio (M) e grande (G) em Acaraú, Ceará.

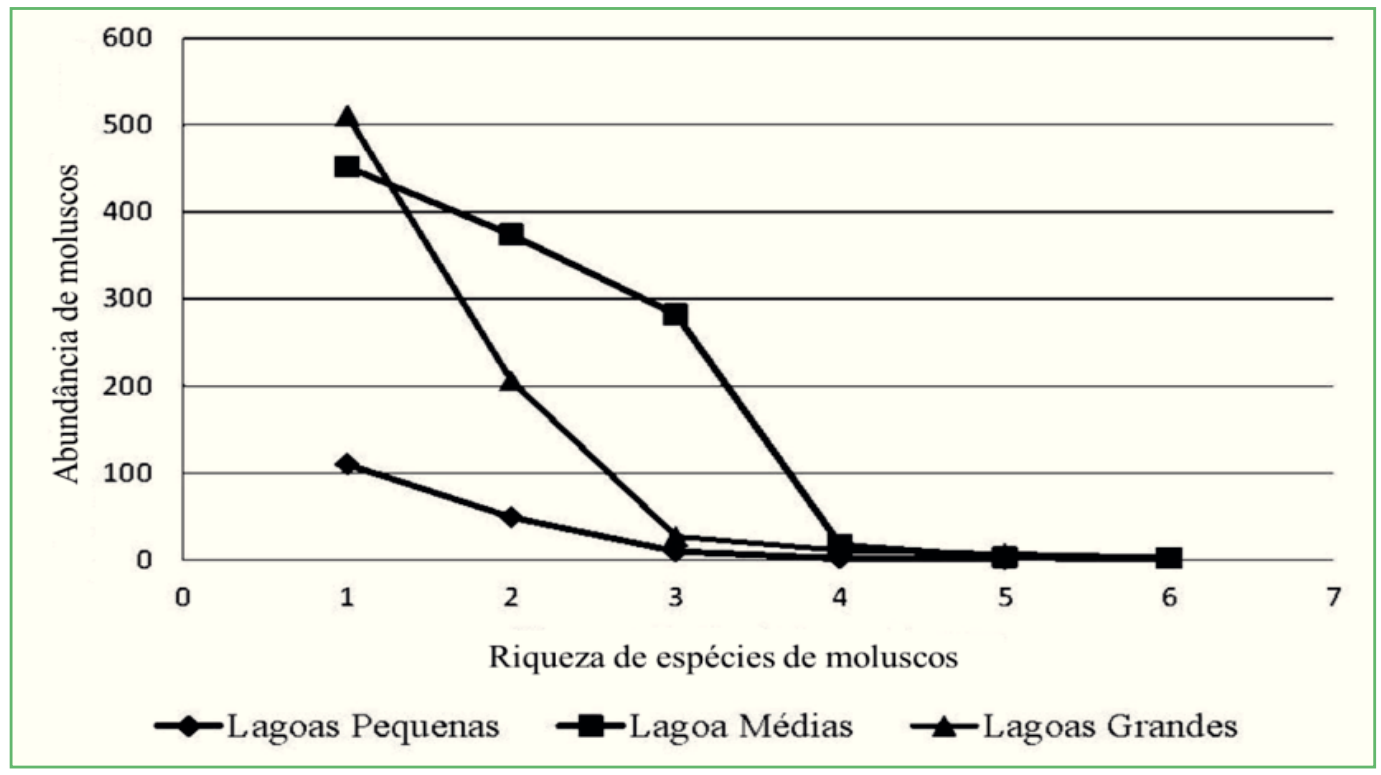


TABELA 3: Índices de diversidade dos moluscos límnicos amostrados nas lagoas de tamanhos pequeno $(\mathrm{P})$, médio $(\mathrm{M})$ e grande $(\mathrm{G})$ em Acaraú, Ceará.

\begin{tabular}{cccc}
\hline Tamanho & Simpson & Shannon & Equabilidade \\
\hline $\mathbf{P}$ & 0,51 & 0,89 & 0,55 \\
$\mathbf{M}$ & 0,67 & 1,16 & 0,65 \\
$\mathbf{G}$ & 0,48 & 0,86 & 0,48 \\
\hline
\end{tabular}

\section{Distância como fator de controle para a dissimilaridade das comunidades de moluscos límnicos}

A análise de agrupamento evidenciou a formação de cinco grupos distintos (Figura 3). Dois grupos apresentaram alta similaridade $(78 \%)$ em relação à estrutura da comunidade de moluscos. O grupo I é formado por Açude Novo e Lagoa Chicó, lagoas pequenas que distam $2.376,3 \mathrm{~m}$, e o grupo II corresponde ao Açude das Piranhas e ao Açude Bal, lagoas grandes que possuem distância de 785,3 m entre elas.

O grupo III apresentou 45\% de similaridade e compreendeu Lagoa Dantas (lagoa média), Açude das Piranhas (lagoa grande) e Açude Bal (lagoa grande). A Lagoa Dantas apresenta 13.340, 6 m de distância do Açude das Piranhas e 14.128,6 m do Açude Bal. A similaridade dessas áreas está relacionada à alta abundância relativa de Thiaridae e Ampullariidae.

O grupo IV reuniu as áreas Açude Novo (lagoa pequena), Lagoa Chicó (lagoa pequena) e Açude Morada Nova (lagoa média). Essas localidades apresentam distâncias medianas, sendo que o Açude novo dista 2.376,3 m da Lagoa Chicó e 2.556,4 m do Açude Morada Nova; no entanto, essas áreas apresentaram similaridade de cerca de $55 \%$ devido à contribuição de Ampullariidae.

FIGURA 3: Dendograma das áreas amostradas, avaliado com uma análise de agrupamento a partir do índice de similaridade, considerando os valores de abundância dos moluscos límnicos nas lagoas amostradas.

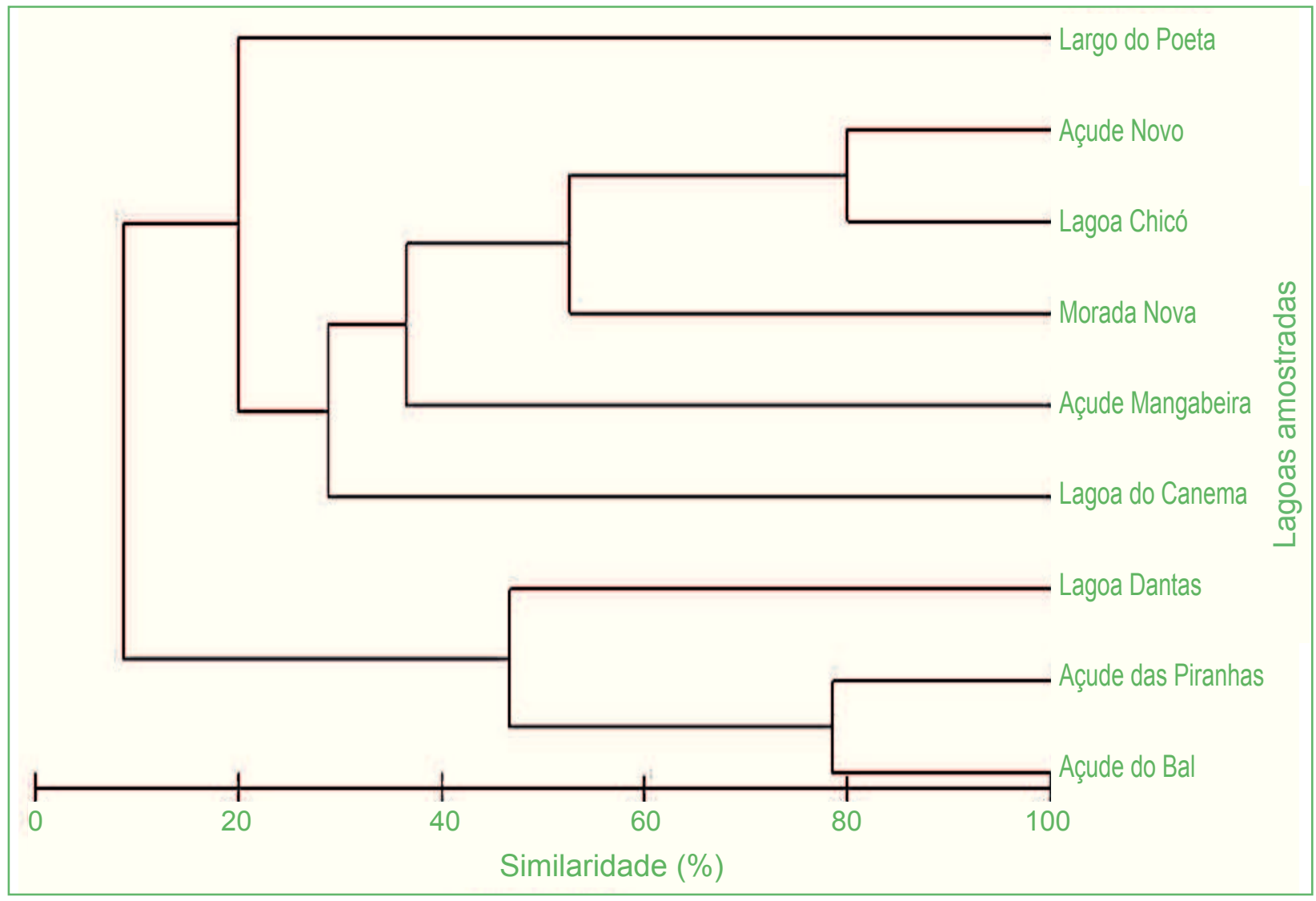


O grupo V agregou Açude Novo (lagoa pequena), Lagoa Chicó (lagoa pequena), Açude Morada Nova (lagoa média), Lagoa do Canema (lagoa média) e Açude Mangabeira (lagoa grande). Nesse agrupamento, a maior distância entre áreas de amostragem foi do Açude Mangabeira, que dista 17.221,7 $\mathrm{m}$ da Lagoa do Canema, 15.055,5 m do Açude Morada Nova, 15.155,5 $\mathrm{m}$ do Açude Novo e 14.774,1 m da Lagoa Chicó. Esses corpos hídricos apresentaram similaridade de 30\%, sendo a abundância de Ampullariidade e Planorbidae responsável por essa semelhança.

O Largo do Poeta (pequena) não formou nenhum agrupamento com o restante das lagoas. Tal padrão se deve à ocorrência de duas famílias Planorbidae e Streptaxidae, sendo que duas espécies, $D$. cimex e $S$. molaris, só ocorreram nesse local.

Assim, os grupos foram formados a partir do grau de similaridade entre as assembleias de moluscos encontrados em cada local e que não apresentaram relação direta com as distâncias entre as lagoas.

\section{Discussão}

A diversidade de gastrópodes de água doce encontrada nas lagoas da cidade de Acaraú no Ceará é semelhante à observada em outros corpos límnicos brasileiros. Por exemplo, Mota (2011), na área do Pesqueiro Itapecerica em São Paulo, registrou seis espécies de seis famílias distintas: $B$. straminea, $M$. tuberculatus, Lymnaea columella, Pomacea lineata, Physa marmorata e Anodontites trapesialis. Vidigal et al. (2005), em trabalho desenvolvido no Rio Doce, Minas Gerais, coletaram os gastrópodes Ancylidae, Planorbidae, do gênero Biomphalaria, Hydrobiidae, Thiaridae, da espécie M. tuberculatus, Physidae, do gênero, Physa, espécimes da família Ampullariidae, com Pomacea, e os Bivalves Sphaeriidae, Corbiculidae e Mycetopodidae.

Pomacea lineata foi encontrada nos nove corpos hídricos utilizados nesse estudo, mostrando sua ampla distribuição nos ambientes límnicos da região. Segundo Thiengo et al. (2004), ampularídeos são encontrados em águas de curso lento e podem suportar ambientes altamente poluídos, como os que recebem esgotos urbanos, o que pode justificar sua dominância nos corpos límnicos estudados em Acaraú. Segundo Pieri (2008), $P$. lineata comporta-se como um consumidor de vegetação, podendo ingerir acidentalmente os ovos de outros moluscos, reduzindo as populações destes ou mesmo eliminando-as, tonando-se dominante no ambiente.

De acordo com Carvalho et al. (2008), os planorbídeos do gênero Biomphalaria registrados para o nordeste são $B$. glabrata, B. tenagophila e B. straminea. No presente trabalho, $B$. straminea foi encontrada em cinco lagoas enquanto $B$. glabrata foi encontrada, em baixa abundância, em apenas uma (Lagoa Morada Nova). Paraense (1972) chama atenção para o fato de que os planorbídeos desse gênero podem ser encontrados em uma grande variedade de ambientes de água doce, parada ou pouco corrente (lagoas, lagos, poços, cisternas, pântanos, rios, riachos, canais de irrigação e outros). $\mathrm{O}$ autor ressalta ainda que Biomphalaria pode tolerar amplas variações nas características físicas, químicas e biológicas do seu ambiente (PARAENSE, 1972).

Biomphalaria straminea está presente em um grande número de municípios brasileiros (1327 munícípios), distribuídos por 24 estados, além do Distrito Federal, com exceção dos estados do Amapá e de Rondônia (CARVALHO et al., 2008). De acordo com Marchiori (1999), na Região Nordeste, a espécie desempenha importante papel na transmissão da esquistossomose como hospedeira intermediária. A espécie já havia sido registrada para região no trabalho desenvolvido por Silveira (2017), que avaliou se esses organismos ofereciam algum risco à população na transmissão da esquistossomose mansônica. Na ocasião, não foram encontrados exemplares infectados.

Biomphalaria glabrata apresenta uma ampla distribuição, sendo encontrada em 16 estados brasileiros e em 806 municípios do país (CARVALHO et al., 2008), porém não havia sido registrada para o estado do Ceará até amostragem no presente trabalho. A espécie é considerada como o hospedeiro intermediário mais eficiente da esquistossomose mansônica nas Américas, sendo responsável por focos ativos (PARAENSE, 1975; REY, 2008). Esses dados devem ser avaliados pelos 
órgãos responsáveis pela saúde pública a fim de evitar a proliferação da esquistossomose na região.

A presença de $M$. tuberculatus foi observada em alta abundância em três lagoas amostradas nesse estudo. Melanoides tuberculatus é um molusco de origem afroasiática introduzido na América Latina em 1960, sendo atualmente distribuído em todos os países da região (VAZ et al., 1986). Acredita-se que sua introdução no país tenha sido por meio do comércio de peixes e plantas ornamentais. Atualmente, sua presença é notificada em vários estados brasileiros (VAZ et al., 1986; FERNANDEZ et al., 2003). Para De Marco (1999), a distribuição dessa espécie por outros sistemas hídricos pode ser facilitada pela presença de pássaros que visitam áreas onde a espécie exótica já está presente. No presente trabalho, $M$. tuberculatus foi encontrado nos corpos hídricos com maiores extensões, possivelmente porque esses locais possuem uma maior área e nichos para abrigar novas espécies pelo processo de migração e mais recursos disponíveis para aves.

Merece destaque também a presença de moluscos da espécie $D$. cimex, coletados em duas áreas em Acaraú:, Largo do Poeta e Açude Morada Nova. No Brasil, é registrada a ocorrência de $D$. cimex para os estados da Bahia (KÓTZIAN; AMARAL, 2013), Minas Gerais (SIMONE, 2006), São Paulo (PEREZ; ARTIGAS, 1969), Paraná (BUENO-SILVA; FISCHER, 2005) e Rio Grande do Sul (FRÓES; LIMA, 1975). O presente trabalho consiste no primeiro registro da espécie para o estado do Ceará e para o município de Acaraú.

Omalonyx sp. foi encontrado exclusivamente no Açude das Piranhas. Arruda et al. (2007) relatou que as lesmas do gênero Omalonyx vivem preferencialmente em ambientes bastante úmidos, muitas vezes alagados, como lagoas, pântanos e margens de outros corpos d'água, em objetos semi-submersos, macrófitas flutuantes e semiaquáticas. No presente estudo, esses organismos foram encontrados na margem vegetada com macrófitas emergentes.

A ocorrência da Gundlachia sp. só foi registrada no Açude das Piranhas. De acordo com Santos (1994), esses moluscos geralmente estão associados a ambientes não poluídos, vivendo preferencialmente aderidos às folhas ou aos talos de plantas aquáticas e em folhas em decomposição, preferindo ambientes lênticos ou com pouca correnteza, tais como lagoas, alagados marginais, remansos de rios ou riachos, brejos, açudes e represas. Esses dados podem indicar o grau de conservação da lagoa amostrada em Acaraú.

Ferrisia sp. foi registrada em três lagoas amostradas. De acordo com Paraense e Santos (2003), esses organismos possuem ampla distribuição nos corpos de água doce, porém, no presente estudo, essa alta frequência não foi observada.

Streptartemon molaris restringiu-se somente a um local e teve baixa ocorrência. De acordo com Simone (2004), o número reduzido da espécie normalmente está relacionado à predação por outro organismo. Cabe ressaltar que essa espécie de molusco é típica da caatinga, justificando sua presença na região.

Com relação ao tamanho dos corpos hídricos, não foram observadas diferenças significativas na riqueza, abundância, diversidade ou estrutura da comunidade das lagoas entre os tamanhos amostrados. A premissa mais difundida na Teoria da Biogeografia de Ilhas para explicar os padrões de ocorrência de espécies é a relação com o tamanho, sendo que a riqueza aumenta progressivamente com o aumento da área (LOPES; CALIMAN, 2008). Ou seja, o tamanho da região pode ser determinante no número de espécies presentes nela, influenciando, por exemplo, o suprimento de colonizadores para uma comunidade. Estudos recentes realizados em poças temporárias e lagos rasos têm sugerido que o tamanho do ecossistema é um fator importante que pode afetar direta e indiretamente mecanismos que determinam os padrões de riqueza de espécies em escalas locais (SCHEFFER et al., 2006).

Os dados obtidos pelo presente estudo não corroboram a proposta da Teoria da Biogeografia de Ilhas, ou pelo menos o modelo não é claro para os moluscos límnicos. No entanto, se observamos individualmente, as lagoas médias tendem a apresentar maior diversidade, riqueza e abundância de moluscos. Tal fato pode ser explicado pela Hipótese do Distúrbio Intermediário, na qual, segundo Cornell e Lawton (1992), a diversidade será maior em comunidades 
com níveis intermediários de perturbação e menor em comunidades com níveis mais baixos (lagoas grandes) ou mais altos de perturbação (lagoas pequenas).

A Hipótese do Distúrbio Intermediário surgiu como mais uma alternativa para explicar a diversidade dentro de comunidades naturais, principalmente a alta diversidade encontrada. Nessa visão, os distúrbios (por exemplo, tempestades, incêndios, inundações), que podem acontecer dentro de várias frequências e intensidades, promovem a mortalidade ou injúrias dos organismos de uma comunidade, e uma maior diversidade é mantida por níveis intermediários de distúrbio (CORNELL; LAWTON, 1992). Com o aumento do intervalo entre os distúrbios, a diversidade também irá aumentar, pois haverá mais tempo para a invasão de diferentes espécies.

O isolamento espacial de comunidades também exerce uma grande influência sobre a dispersão de organismos entre diferentes comunidades, tendo assim um efeito sobre a diversidade. Segundo a Teoria de Biogeografia de Ilhas, um maior grau de isolamento reduz a taxa de colonização de novos indivíduos e espécies para uma comunidade, diminuindo, portanto a riqueza de espécies. Hubbell (2001) também prediz que a similaridade de espécies diminui com o aumento da distância entre locais. Entretanto, neste estudo, não foi observada similaridade entre as lagoas próximas. A baixa riqueza de espécies foi preponderante para similaridade entre as lagoas nesse estudo.

A semelhança na composição dos moluscos entre as lagoas também pode ser explicada pela facilidade de dispersão por toda a área, inclusive devido às enchentes dos corpos hídricos, que podem interligar alguns dos corpos de água amostrados, sendo o tempo de isolamento não suficiente para determinar diferenças nas assembleias de moluscos. Por exemplo, o Açude das Piranhas, nos períodos chuvoso, se conecta com o Açude Bal e a Lagoa Chicó, favorecendo a dispersão de organismos entre esses ambientes, corroborando a similaridade das espécies entre os locais, mesmo em períodos de estiagem.

Assim, o presente trabalho conclui que a composição de espécies de moluscos límnicos em
Acaraú é semelhante à encontrada em outros corpos de água doce lênticos no Brasil. E faz um primeiro registro para o estado do Ceará de B. glabrata e $D$. cimex, ambos pertencentes à família Planorbidae. $\mathrm{O}$ tamanho dos corpos hídricos não exerceu influência significativa na comunidade de moluscos. Entretanto, há uma tendência de maior riqueza, abundância e diversidade em lagoas médias, o que pode ser resultante de distúrbios intermediários. Também não foram observadas similaridades na estrutura da comunidade de moluscos entre corpos de água próximos, o que pode ser explicado pela facilidade de dispersão das espécies por toda a área, devido às enchentes dos corpos hídricos em períodos mais chuvosos.

\section{Agradecimentos}

Ao Instituto Federal de Educação, Ciência e Tecnologia do Ceará - Campus de Acaraú, pela logística. À equipe do laboratório Ecologia de Manguezais (ECOMANGUE), pelo auxílio nas coletas. Aos avaliadores A. L R. Brandão e I. H. C. V. Silva, por suas contribuições ao manuscrito.

\section{Referências}

AMARAL, A. C.; RIZZO, A. E.; ARRUdA, E. P. Manual de identificação dos invertebrados marinhos da região SudesteSul do Brasil. Vol. 1. São Paulo: Ed Universidade de São Paulo, 2005. 287 p.

ARRUDA, J. O. Sistemática e ecologia de espécies de Omalonyx (Mollusca, Gastropoda, Succineidae) no Estado do Rio Grande do Sul. 2007. 47 f. Dissertação (Mestrado em Zoologia) - Pontifícia Universidade Católica do Rio Grande do Sul, Porto Alegre. 2007.

BUENO-SILVA, M.; FISCHER, M. L. Dinâmica populacional de Drepanotrema cimex (Moricand, 1839) (Mollusca: Basommatophora: Planorbidae) no Parque Barigüi, Curitiba, Paraná, Brasil. Biotemas, Florianopolis, v. 18, n. 2, p. 129-141, 2005.

CARPENTER, S. R. Trophiccascades, nutrients, andlake productivity: whole-lake experiments.Ecological Monographs, Ithaca, v. 71, n. 5, p. 163-186, 2001.

CARVAlHO, J. B.; AlMEIDA, E. A. B. Áreas de endemismo. In: CARVALHO, C. J. B. de; LAVINA, E. L.; FAUTH, F. (Ed). Biogeografia da América do Sul: padrões e processos. São Paulo: Roca. 2011. p. 41-51.

CARVALHO, O. S.; AMARAL, R S.; DUTRA, L V.; SCHOLTE, R. G. C.; GUERRA, M. A. M. Distribuição espacial de Biomphalaria glabrata, B. straminea e $B$. tenagophila, 
hospedeiros intermediários de Schistosoma mansoni no Brasil. In: CARVALHO, O. S.; COELHO, P. M. Z.; LENZI, H. L. (Org.). Schistosoma mansoni e esquistossomose. Rio de Janeiro: Editora FIOCRUZ, 2008. p. 393-418.

CLARKE, K. R.; GORLEY, R. M. Primer v6: user manual/ tutorial. PRIMER-E, Plymouth change in marine communities: an approach to statistical analyses and interpretation. 2 ed. Plymouth: PRIMER-E, 2006.

CLIMATE-DATA.ORG. Dados climáticos para cidades mundiais. Disponível em: <https://pt.climate-data.org/location/44118/>. Acesso em: 14 nov. 2017.

CORNELL, H. V; LAWTON, J. H. Species interactions, local and regional processes, and limits to the richness of ecological communities: a theoretical perspective. Journal of Animal Ecology, London, v. 61, p. 1-12, 1992.

DE MARCO, P. J. Invasion by the introduced aquatic snail Melanoides turbeculata (Müller, 1774) (Gastropoda: Prosobranchia: Thiaridae) of the Rio Doce State Park, Minas Gerais, Brazil. Studies on Neotropical Fauna and Environment, Abingdon, v. 34, p. 186-189, 1999.

DE MEESTER, L.; DECLERCK, S.; STOKS, R.; LOUETTE, G.; VAN DE MEUTTER, F.; DE BIE, T.; MICHELS, E.; BRENDONCK, L. Ponds and pools as model systems in conservation biology, ecology and evolutionary biology. Aquatic Conservation: Marine and Freshwater Ecosystems, Malden, v. 15, p. 715-725, 2005.

DODSON, S. I. Predicting crustacean zooplankton species richness. Limnology and Oceanography, Newfoundland, v. 37, p. 848-856, 1992.

DRÜGG-HAHN, S. Potencial de atuação de Heleobia piscium (Orbigny, 1835) (Molusca, Hydrobiidae) como hospedeiro intermediário de Nocotylidae (Platyhelminthes, Trematoda), no RS. 1997. 78 f. Dissertação (Mestrado em Biologia Animal) Universidade Federal do Rio Grande do Sul, Porto Alegre. 1997.

FERNANDEZ, M. A.; THIENGO, S. C; SIMONE, L. R. Distribution of the introduced freshwater snail Melanoides tuberculatus (Gastropoda: Thiaridae) in Brazil. The Nautilus, Sanibel, v. 117, p. 78-82, 2003.

FRANÇA, R.S,; SURIANI, A. L.; ROCHA, O. Composição das espécies de moluscos bentônicos nos reservatórios do baixo Tietê (São Paulo, Brasil), com uma avaliação dos impactos causados pelas espécies exóticas invasoras. Revista Brasileira de Zoologia, Curitiba, v. 24, n. 1, p. 41-51, 2007.

FRÓES, O. M.; LIMA, D. F. Contribuição ao conhecimento da biogeografia, sistemática e ecologia dos planorbídeos (Gastropoda, Planorbidae) do Rio Grande do Sul. Iheringia, Porto Alegre, v. 47, p. 67-72, 1975.

GILLER, P. S.; HILLEBRAND, H.; BERNINGER, U-G.; GESSNER, M. O.; HAWKINS, S.; INCHAUSTI, P.; INGLIS, C.; LESLIE, H.; MALMQVIST, B.; MONAGHAN, M. T.; MORIN, P. J.; O'MULLAN, G. Biodiversity effects on ecosystem functioning: emerging issues and their experimental test inaquatic environments. Oikos, Copenhagen, v. 104, n. 4, p. 423-436, 2004.

GILLUNG, J. P. Biogeografia: a história da vida na Terra. Revista da Biologia, São Paulo, v. especial, p. 1-5, 2011.

HUBBELL, S. P. The united neutral theory of biodiversity and biogeography. Princeton: University Press, 2001. 396 p.
KARDONG, K. V. Vertebrados - Anatomia Comparada, Função e Evolução. 5. ed. São Paulo: Editora Roca, 2011. 913 p.

KÓTZIAN, C. B.; AMARAL, M. B. Diversity and distribution of mollusks along the Contas River in a tropical semiarid region (Caatinga), Northesatern Brazil. Biota Neotropica, Campinas, v. 13, n. 4, p. 299-314, 2013.

LOPES, P. M.; CALIMAN, A. A contribuição de ecossistemas lênticos para o entendimento da importância de processos regionais e locais sobre padrões geográficos de biodiversidade. Rio de Janeiro: Publicação da Sociedade Brasileira de Limnologia, 2008. 29 p.

LOPES-PITONI, V. L. Acompanhamento e avaliação da flora e fauna do Parque COPESUL de Proteção Ambiental, município de Triunfo, RS. In: Porto Alegre: Fundação Zoobotânica do Rio Grande do Sul, MCN. Relatório técnico. 2001. 198 p.

LUDWIG, A. J.; REYNOLDS, F. J. Statistical ecology: a primer a methods and computing. New York: John Wiley and Sons, 1988.

MACARTHUR, R. H.; WILSON, E. O. The Theory of Island Biogeography. Princeton: University Press, 1967. 203 p.

MARCHIORI, C. H. Primeira ocorrência de Biomphalaria straminea no Sul Goiano, Brasil. Revista de Saúde Pública, São Paulo, v. 33, n. 6, p. 622-623, 1999.

MOTA, D. J. G. Levantamento da malacofauna límnica na área do Pesqueiro Itapecerica, Itapecerica da Serra /São Paulo. 2011. 162 f. Dissertação (Mestrado Ciências) - Coordenadoria de Controle de Doenças da Secretaria do Estado da Saúde de São Paulo, São Paulo. 2011.

PARAENSE, W. L. Fauna planorbídica do Brasil. In: LACAZ, C. S.; BARUZZI, S. Jr. (Ed.). Introdução à geografia médica do Brasil. São Paulo: Edgard Blucher, 1972. p. 213-239.

PARAENSE, W. L. Estado atual da sistemática dos planorbídeos brasileiros. Arquivos Museu Nacional do Rio de Janeiro, Rio de Janeiro, v. 55, p. 105-128, 1975.

PARAENSE, W. L.; SANTOS, S. B. A bird's eye survey of Central American planorbid molluscs. Memórias do Instituto Oswaldo Cruz, Rio de Janeiro, v. 98, n. 1, p. 51-67, 2003.

PEREZ, M. D.; ARTIGAS, P. T. Contribuição ao levantamento da carta planorbídica do estado de São Paulo. Pesquisa de focos com formas evolutivas do Schistosoma mansoni. II. Município de Peruíbe (Litoral Sul do estado de São Paulo, Brasil). Revista de Saúde Pública, São Paulo, v. 3, n. 2, p. 149-152, 1969.

PIERI, O. S. Aspectos ecológicos. In: Vigilância e controle de moluscos de importância epidemiológica - Diretrizes técnicas: Programa de Vigilância e Controle da Esquistossomose. 2. ed. Brasília: Ministério da Saúde, 2008. 178 p.

POOLE, R. W. Introduction to quantitative ecology. Tokyo: Mc Graw-Hill, 1974. 532 p.

RECODER, R. S. Biogeografia baseada em eventos: uma introdução. Revista da Biologia, São Paulo, v. especial Biogeografia, p. 18-25, 2011.

REY L. Parasitologia: parasitos e doenças parasitárias do homem nos trópicos ocidentais. 4 ed. Rio de Janeiro: Guanabara Koogan, 2008. 883 p.

SANTOS, S. B. Gundlachia dutrae n.sp. from northeastern Brazil (Mollusca: Basommatophora: Ancylidae). Memórias do Instituto Oswaldo Cruz, Rio de Janeiro, v. 89, p. 153-160, 1994. 
SANTOS, S. B.; RODRIGUES, C. L.; NUNES, G. K. M.; BARBOSA, A. B.; LACERDA, L. E. M.; MIYAHIRA, I. C.; VIANA, T. A.; OLIVEIRA, J. L.; FONSECA, F. C.; SILVA, P. S. C. Estado do conhecimento da fauna de invertebrados não-marinhos da Ilha Grande (Angra dos Reis, RJ). Oecologia Australis, Rio de Janeiro, v. 14, p. 504-549, 2010.

SCHEFFER, M.; VAN GEEST, G. J.; ZIMMER, K.; JEPPESEN, E.; SONDERGAARD, M.; BUTLER, M. G.; HANSON, M. A.; DECLERCK, S. DE MEESTER, L. Small habitat size and isolation can promote species richness: second-order effects on biodiversity in shallow lakes and ponds. Oikos, Copenhagen, v. 112, p. $227-$ 231, 2006.

SILVEIRA, E. P. R. Caracterização de planorbídeos do gênero Biomphalaria (Gastropoda Mollusca) encontrados em sistemas límnicos do munícipio de Acaraú - $\mathrm{CE}$ análise de possíveis focos de transmissão de esquistossomose mansônica. $2017.50 \mathrm{f}$. Trabalho de Conclusão de Curso (Graduação em Licenciatura em Ciências Biológicas) - Instituto Federal de Educação, Ciência e Tecnologia do Ceará, Acaraú. 2017.

SIMONE L. R. L. Comparative morphology and phylogeny of representatives of the superfamilies of Architaenioglossans and the Annulariidae (Mollusca, Caenogastropoda). Arquivos do Museu Nacional, Rio de Janeiro, v. 62, p. 387-504, 2004.
SIMONE, L. R. L. Land and freshwater molluscs of Brazil. São Paulo: EGB/FAPESP, 2006. 390 p.

SIMONE, L. R. L. Histórico da malacologia no Brasil. Revista de Biología Tropical, San José, v. 51, p. 139-147, 2003.

SRIVASTAVA, D. S.; KOLASA, J.; BENGTSSON, J.; GONZALEZ, A.; LAWLER, S. P.; MILLER, T. E.; MUNGUIA, P.; ROMANUK, T.; SCHNEIDER, D. C.; TRZCINSKI, M. K. Are natural microcosms useful model systems for ecology? Trends in Ecology and Evolution, Cambridge, v. 19, p. 379-384, 2004.

THIENGO, S. C.; MATTOS, A. C.; BOAVENTURA, M. F.; LOUREIRO, M. S.; SANTOS, S. B.; FERNANDEZ, M. A. Freshwater snails and schistosomiasis mansoni in the State of Rio de Janeiro, Brazil: V - Norte Fluminense Mesoregion. Memórias do Instituto Oswaldo Cruz, Rio de Janeiro, v. 99, p. 99-103, 2004.

VAZ, F. V.; TELES, H. M. S.; CORREA, M. A.; LEITE, S. P. S. Ocorrência no Brasil de Thiara (Melanoides) tuberculata (O.F, Muller, 1774) (Gastropoda, Prosobranchia), primeiro hospedeiro intermediário de Clonorchis sinensis (Cobbold, 1875) (Trematoda, Plathyhelmintes). Revista de Saúde Pública, São Paulo, v. 20, n. 4, p. 318-322, 1986.

VIDIGAL, T. H. D. A.; MARQUES, M. G. S. M.; LIMA, H. P.; BARBOSA, A. R. Gastrópodes e bivalves límnicos do trecho médio da bacia do Rio Doce, Minas Gerais, Brasil. Lundiana, Belo Horizonte, v. 6 (supplement), p. 67-76, 2005. 